\title{
EX-MET study: exercise in prevention on of metabolic syndrome - a randomized multicenter trial: rational and design
}

Arnt Erik Tjønna ${ }^{1,9,10^{*}}$, Joyce S. Ramos ${ }^{2}$, Axel Pressler ${ }^{3}$, Martin Halle ${ }^{3,8}$, Klaus Jungbluth $^{4}$, Erika Ermacora ${ }^{4}$, Øyvind Salvesen ${ }^{5}$, Jhennyfer Rodrigues ${ }^{6}$, Carlos Roberto Bueno $\mathrm{Jr}^{6}$, Peter Scott Munk', Jeff Coombes ${ }^{2}$ and Ulrik Wisløff ${ }^{1,2}$

\begin{abstract}
Background: Metabolic syndrome substantially increases risk of cardiovascular events. It is therefore imperative to develop or optimize ways to prevent or attenuate this condition. Exercise training has been long recognized as a cornerstone therapy for reducing individual cardiovascular risk factors constituting the metabolic syndrome. However, the optimal exercise dose and its feasibility in a real world setting has yet to be established.

The primary objective of this randomized trial is to investigate the effects of different volumes of aerobic interval training (AIT) compared to the current exercise guideline of moderate-intensity continuous training (MICT) on the composite number of cardiovascular disease risk factors constituting the metabolic syndrome after a 16 week, 1-year, and 3-year follow-up.
\end{abstract}

Methods: This is a randomized international multi-center trial including men and women aged $\geq 30$ years diagnosed with the metabolic syndrome according to the International Diabetes Federation criteria. Recruitment began in August 2012 and concluded in December 2016. This trial consists of supervised and unsupervised phases to evaluate the efficacy and feasibility of different exercise doses on the metabolic syndrome in a real world setting. This study aims to include and randomize 465 participants to 3 years of one of the following training groups: i) 3 times/week of $4 \times 4$ min AlT at 85-95\% peak heart rate (HRpeak); ii) 3 times/week of $1 \times 4$ min AlT at 85-95\% HRpeak; or iii) 5-7 times/week of $\geq 30$ min MICT at 60-70\% HRpeak. Clinical examinations, physical tests and questionnaires are administered to all participants during all testing time points (baseline, 16 weeks and after 1-, and 3-years).

Discussion: This multi-center international trial indeed aims to ease the burden in healthcare/economic cost arising from treating end-stage CVD related conditions such as stroke and myocardial infarction, that could eventually emerge from the metabolic syndrome condition.

Trial registration: Clinical registration number: NCT01676870, ClinicalTrials.gov (August 31, 2012).

Keywords: Exercise training, Metabolic syndrome, Cardiovascular disease

\footnotetext{
* Correspondence: arnt.e.tjonna@ntnu.no

${ }^{1}$ K.G. Jebsen Center of Exercise in Medicine at Department of Circulation and

Medical Imaging, Faculty of Medicine, Norwegian University of Science and

Technology, Trondheim, Norway

${ }^{9}$ Clinic of Cardiology, St. Olavs Hospital, Trondheim, Norway

Full list of author information is available at the end of the article
} 


\section{Background}

Several lines of evidence suggest that endurance training improves cardiovascular function $[1,2]$. Improvements in maximal work capacity are accompanied by several central and peripheral cardiovascular adaptations that are regarded as the signature of the trained state. These adaptive changes include increased maximal oxygen uptake, improved cardiac- and endothelial function, improved lipid profile and increased abundance of capillaries, mitochondria and metabolic enzymes.

Several of these factors are related to the components of metabolic syndrome, including central obesity, hypertriglyceridemia, low HDL cholesterol, hypertension and raised fasting plasma glucose [3]. Currently, about $25 \%$ of the US adult population is classified as having the metabolic syndrome, and the prevalence is expected to increase in parallel to the pandemic of obesity and overweight, which affects about 312 million and 1.1 billion people worldwide, respectively [4, 5]. European numbers seems to be similar to that observed in the US. The European Group for the Study of Insulin Resistance (EGIR) reported that among non-diabetic individuals, the frequency of the metabolic syndrome varied from 7 to $36 \%$ for men and between 5 to $22 \%$ in women aged 40 to 55 years [6]. It is well established that the risk for premature cardiovascular death is increased in individuals with the metabolic syndrome [7, 8]. Data from Katzmarzyk et al. [2] demonstrate a relative risk of 1.89 (95\% CI: 1.36-2.60) for premature cardiovascular mortality in men with metabolic syndrome compared to healthy men. In line with this, combining several factors associated with cardiovascular disease (CVD) gives a more powerful predictor of premature CVD death than assessing separate effects of each factor [4]. Exercise training may reverse most of the risk factors of the syndrome and also protects against premature cardiovascular death [1, 2, 9]. However, the recommendation of moderate physical activity does not seem to prevent the syndrome of reaching pandemic levels and the optimal training regimen remains to be defined.

Today we have good indications that exercise training is an effective contributor for reducing risk factors related to metabolic syndrome [1, 2], but no sufficiently powered, randomized controlled study have documented the contribution of exercise training in the reversal of metabolic syndrome.

\section{Aims}

Primary aim of EX-MET

- The primary aim of the study is to compare the efficacy of traditional training (today's guideline according to the American College of Sports Medicine [ACSM], vigorously or moderate exercise) and different volumes of aerobic interval training (1-AIT vs. 4-AIT) in reducing risk factors constituting the metabolic syndrome in a real world setting.

\section{Secondary aims of EX-MET}

- The secondary objectives are to compare the efficacy of traditional moderate training (ACSM guideline) and different volumes of aerobic interval training (1-AIT vs. 4-AIT) in improving aerobic capacity, cardiovascular function, skeletal muscle contractile function, skeletal muscle energy metabolism, left ventricle systolic and diastolic function at rest and right ventricular function.

\section{Methods}

Design

Participants with the metabolic syndrome defined according to the IDF-criteria [3] are included in this trial. Patients (male and female age $\geq 30$ ) are randomized and stratified (age, gender and center) into the $4 \times 4 \mathrm{~min}$ aerobic interval training (4-AIT, vigorously exercise according to today's guidelines [ACSM], three times a week), 1x4min aerobic interval training (1-AIT, three times a week) and moderate-intensity continuous training (MICT, according to ACSM guidelines) groups at a 1:1:1 ratio. This trial consists of two phases. The first phase requires the participants to attend two supervised training sessions with an exercise physiologist per week for 16 weeks, with the residual sessions performed in an unsupervised environment. Participants then enter the second phase of the trial and are instructed to proceed with the same exercise program from the previous 16-week intervention, but in an unsupervised manner. During this phase, they are required to attend a supervised exercise session each month, until the one-year follow-up, and every six months until the 3-year end-point. The participants are tested at baseline, after 16 weeks, and at one-, and three-year follow-up (Fig. 1). The Unit for Applied Clinical Research at the Norwegian University of Science and Technology developed the randomization procedure to ensure impartial assignments. After randomization, participants receive verbal and written information about their intervention. EX-MET has been approved by the Regional Committee for Medical Research Ethics (REK 2011/2150), and was registered in the clinical trials registry in August 2012 (ClinicalTrials.gov, Identifier: NCT01676870). Oral and written informed consent is obtained from all participants before inclusion. Participation in the main study is not influenced by willingness to participate in sub-studies. All sub-studies must have approval from the Regional Committee for Medical Research Ethics and studies will not involve interventions that are in conflict with the main study. Exclusion criteria are unstable angina, recent myocardial 


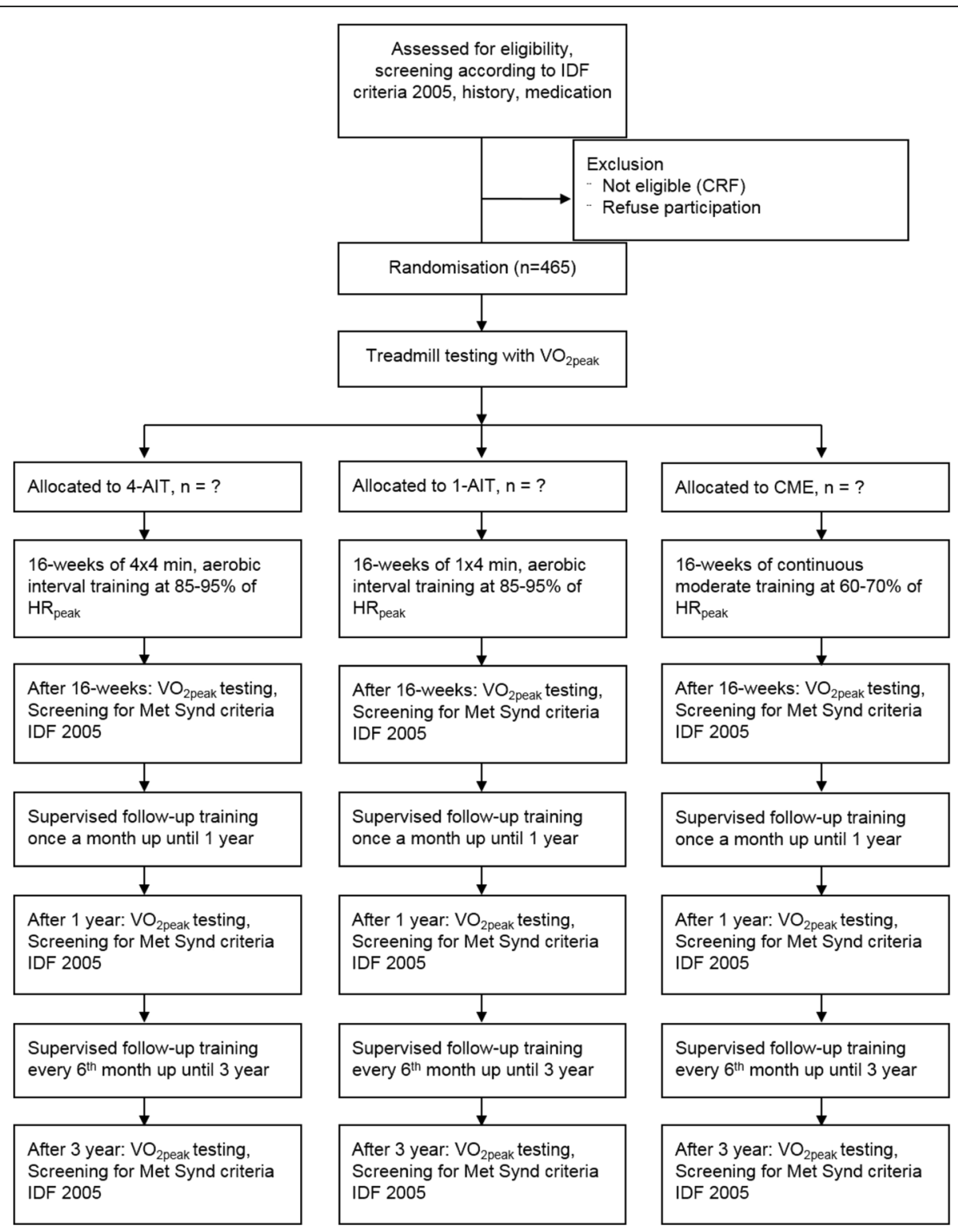

Fig. 1 Study design

infarction (within the last four weeks), uncompensated heart failure, severe valvular illness, pulmonary disease, uncontrolled hypertension, kidney failure, orthopedic/neurological limitations, cardiomyopathy, and planned operations during the research period, reluctant to sign the consent form, drug or alcohol abuse or participants in a parallel study.

\section{Project organization}

The Norwegian University of Science and Technology is the coordinating center of this multi-center trial. This center coordinates and maintains the randomization database, clinical report forms, and adverse effect registry. A steering committee has been appointed to dictate resolutions for major scientific and ethical questions. This committee developed the study protocol and is responsible for data collection, management, and publications derived from this study. Inclusion of participants started in August 2012 and is estimated to finish by the end of 2016. Each center is expected to include at least 50 patients, while the larger sites $\geq 100$.

\section{Participating centers}

Six centers with local coordinators will include patients:

1. Trondheim (Norway) - Arnt Erik Tjønna

2. Brisbane (Australia) - Jeff Coombes

3. Stavanger (Norway) - Peter Scott Munk 
4. Guayaquil (Equador) - Klaus Jungbluth

5. Munich (Germany) - Martin Halle

6. Sao Paulo (Brazil) - Carlos Roberto Bueno Jr.

\section{Ethics considerations}

The risk of exercise is considered small. However, the risk of complications/death is higher during and immediately following training/testing [10]. Nevertheless, the health benefits from exercise training are sufficiently high and are considered less harmful than inactivity. Providing sufficient clear information is a high priority, so that participants feel that they know what consenting entails.

\section{Examinations}

The following examinations are performed at time points indicated above and are described in more detail in our standard operating procedures (Appendix 1). Briefly, blood samples are obtained from an arm vein after a 12-h overnight fast. Triglycerides (TG), glucose, insulin, highdensity lipoprotein-cholesterol (HDL-C), low-density lipoprotein-cholesterol (LDL-C), total cholesterol, Creactive protein $(\mathrm{CRP})$, glycosylated hemoglobin $\left(\mathrm{HbA}_{1 \mathrm{c}}\right)$, and $\mathrm{C}$-peptide are measured using standard procedures at respective hospitals or centers with high standard of accuracy. Serum and EDTA plasma are centrifuged at $2500 \mathrm{rpm}$ for $10 \mathrm{~min}$ at $20^{\circ} \mathrm{C}$. Aliquots are stored at $-80^{\circ} \mathrm{C}$ in case new blood markers will be analyzed later. In addition, whole blood from an EDTA tube is taken and stored (at Regional Biobank) at $-80^{\circ} \mathrm{C}$ for later analysis of DNA and other blood markers. Blood pressure is measured after a 12-h overnight fast and following $15 \mathrm{~min}$ of seated quiet rest. Testing of cardiorespiratory fitness (peak oxygen uptake, $\dot{\mathrm{V}} \mathrm{O}_{2 \text { peak }}$ ) is performed on a treadmill or cycle ergometers identical to that in previous studies in our group [11]. Ergometer selected for testing is dependent upon preferred type of exercise during the intervention, as well as physical limitations presented by participants. Participants with previous heart diseases are tested under electrocardiograph (ECG) monitoring, and the ACC/AHA-guidelines for exercise testing of patients with known cardiovascular disease is followed [12]. Body composition and weight are measured using bioelectrical impedance (Inbody 720, BIOSPACE, Seoul, Korea). Physical activity is measured via SenseWear Armband activity monitor (BodyMedia 7, Pittsburgh, PA, USA) or by Actigraph (GT3X, Manufactering Technology Inc., Florida, USA). After the clinical test at the hospital, all participants are given either SenseWear or Actigraph monitor. The participants are instructed to wear it for seven days continuously (24 h), except when in contact with water (shower/bath/swim). Novel genetic biomarkers (DNA and RNA) will be analyzed using highthroughput OMICS technology (genotyping or exome sequencing, DNA methylation, RNA sequencing, microRNA screening, MS-based proteomics, and MR metabolomics) using validated methods established at the Norwegian University of Science and Technology's Genomics, Proteomics and Metabolomics Core Facilities. Health economic analysis will be calculated as the sum of hospital cost (inpatient, day care, and outpatient care), use of general physician, and primary health care (rehabilitation, nursing homes, home care etc.). Five different questionnaires are used in this study: Questionnaire 1 consisted of questions about specific aspects of physical activity history. The short form health survey SF-8 (1-wk recall version) is used to describe quality of life and chronic pain (Q3). The questions from Q1, Q2 and Q3 are the same questions that have been used in the NordTrøndelag Health Study (HUNT) [13-15]. Medications were monitored throughout the study.

\section{Sample size and statistics}

According to estimates based on data from previous studies, $[16,17]$ a total of 465 patients randomized 1:1:1 to the three interventions gives $80 \%$ power to detect an effect of 4-AIT compared to 1 -AIT/CME of $51 \%$ recovery versus $37 \%$ recovery from metabolic syndrome at the $5 \%$ significance level. These calculations were based on the following assumptions: The primary endpoint is reduction in the composite number of cardiovascular risk factors constituting the metabolic syndrome (IDF) [3], as a change from baseline to one year follow-up.

\section{Training interventions \\ Aerobic interval training}

Participants that are randomized into the aerobic interval training groups (4-AIT and 1-AIT) perform a total of three training sessions per week, for 16 weeks, during the first phase of the trial. Two training sessions are performed in the hosting research institution with an exercise physiologist, and one training session in an unsupervised environment. During the second phase of the trial, all three sessions per week are performed in their own time at home, outdoor or in a gym. Participants are provided with a training log to record all unsupervised training sessions. All training sessions involve activities using large muscle mass. Supervised training sessions are generally performed on a cycle or treadmill ergometer (self-selected). Unsupervised sessions consist of outdoor/indoor pursuits involving different activities such as running, walking, cycling, rowing, swimming, and skiing, performed in the prescribed AIT format. The 4-AIT and 1-AIT groups exercise for 38 and $17 \mathrm{~min}$ per session, respectively. Both the AIT protocols include a 10-min warm-up and a 3-min cool-down at $60-70 \%$ peak heart rate (HRpeak) or at a rate of perceived exertion [RPE] of 11-13 on a Borg scale. Following the 10min warm-up, a 4-min interval is performed at $85-95 \%$ HRpeak (RPE at 15-17 on Borg scale). This is repeated 
4 times in the 4-AIT group, separated by 3 min of active recovery at $50-70 \%$ HRpeak (Fig. 2a). The 1-AIT group only performs one 4-min interval at the same intensity and immediately proceeds to the cool-down period (Fig. 2b). The target heart rate should be reached within two minutes of the 4-min interval period for both volumes of AIT (1-AIT and 4-AIT). Heart rate is monitored (Polar electro, Kempele, Finland) during all supervised exercise sessions to ensure participants are reaching the prescribed target heart rate. Participants are encouraged to purchase their own heart rate monitor to keep track of their own training during the unsupervised sessions. Otherwise, they are instructed to use the Borg 6-20 scale (rating of perceived exertion) to serve as a guide towards the exercise intensity achieved [18]. HR and RPE during all training (unsupervised and supervised) sessions are monitored and recorded.

\section{Moderate-intensity continuous training}

Participants randomized into the MICT group perform 5-7 training sessions per week to conform to the current exercise guideline put forth by the American College of Sports Medicine (ACSM). Two sessions are supervised in an exercise laboratory by an exercise physiologist, and the rest are completed in an unsupervised environment. Training modes for both the supervised and unsupervised sessions are the same as AIT. The MICT group trains continuously for $\geq 30 \mathrm{~min}$ at a target intensity of $60-70 \%$ HRpeak (RPE of $11-13$ on
Borg scale) with no signs of shortness of breath (Fig. 2c). $\mathrm{RPE}$ and HR are monitored and recorded for all training sessions as indicated above.

\section{Discussion}

To our knowledge, EX-MET is the first randomized trial to investigate the impact of different volumes of AIT (1AIT and 4-AIT) and MICT on the treatment of metabolic syndrome in individuals from different ethnic groups. This trial aims to extend previous small studies ( $n=27$ to 40 ) without a follow-up period that have examined the efficacy of high volume-AIT compared to an iso-caloric MICT on traditional and novel cardiovascular disease (CVD) risk factors [11, 16, 19, 20]. Recent metaanalyses suggest that the greater tendency of high volume-AIT (4-AIT) relative to the traditional MICT to reduce risk of CVD and mortality could be attributable to its greater ability to improve cardiorespiratory fitness [21] and vascular function [22] in clinical populations. Specifically, we have demonstrated a greater impact of 4AIT compared to an isocaloric MICT in attenuating individual components of the metabolic syndrome, as well as improving cardiorespiratory fitness, vascular function, and mitochondrial function in a small pilot study incorporating 32 individuals diagnosed with metabolic syndrome [16]. We also recently demonstrated that low volumeAIT (1-AIT) is equally as effective as 4-AIT in improving cardiorespiratory fitness in short term in overweight individuals [23]. Thus since cardiorespiratory fitness has been

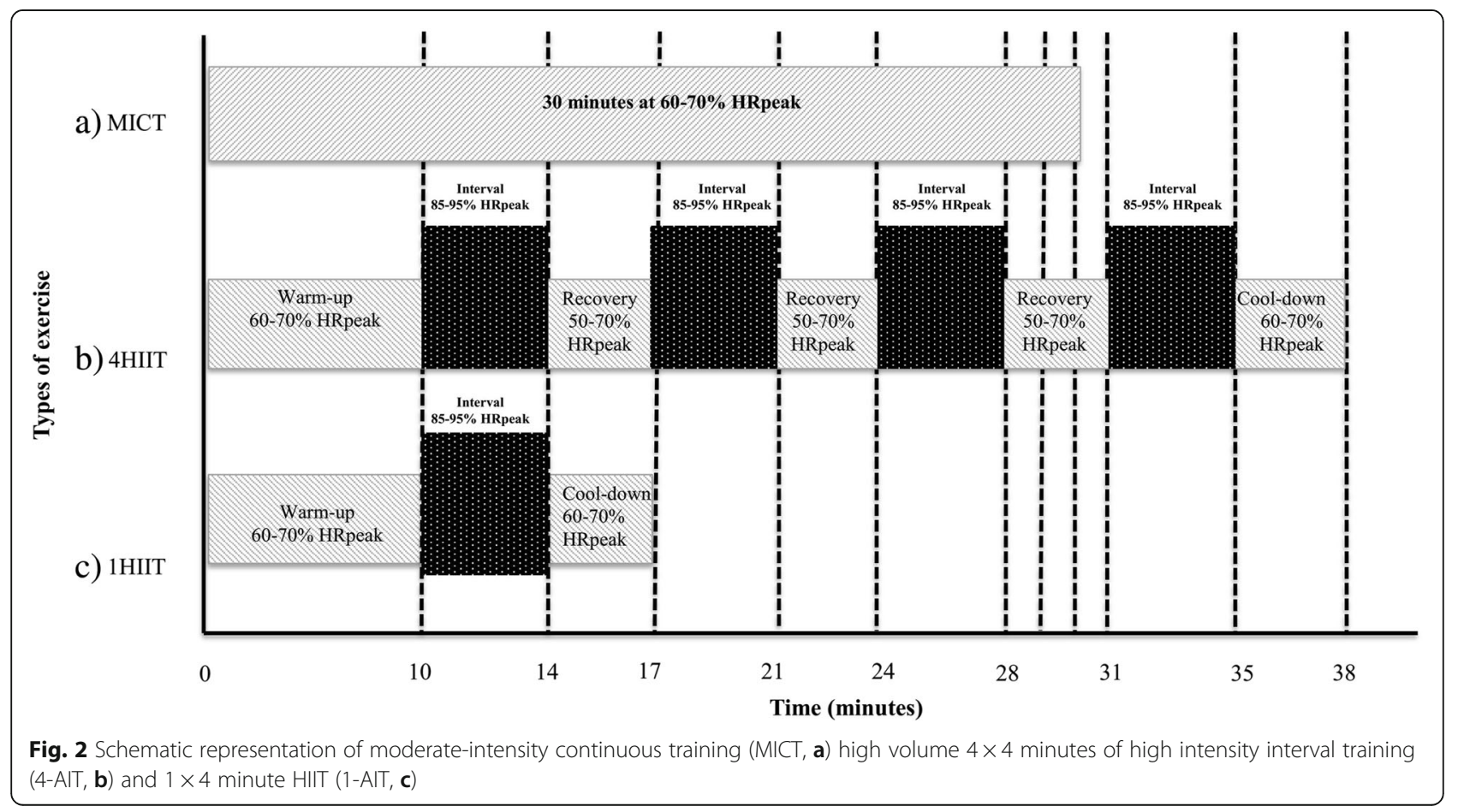


shown to be an antidote towards different CVD risk factors associated with the metabolic syndrome [24], and that 'lack of time' has been the most reported barrier to exercise [25], it was logical for us to investigate the impact of low-volume AIT (1-AIT) compared to high-volume dosages of high- (4-AIT) and moderate-intensity exercise (MICT) prescribed according to the current exercise guideline (ACSM). This could help decipher an optimal or alternative, but effective, exercise prescription that we suspect most individuals with metabolic syndrome would better adhere or comply with, in attempt to ease the economic burden of this pandemic.

It is noteworthy that most studies that have investigated the impact of AIT compared to MICT on several factors affecting health in a diverse clinical population have only been from a supervised laboratory setting, conducted over a short-term period (12-16 weeks) [21, 22, 26]. Thus, although most studies revealed a greater tendency for AIT to improve CVD risk factors associated with the metabolic syndrome relative to the current exercise guideline, it is still of question on whether individuals would be able to perform this type of exercise in an unsupervised environment for a long-term period. This multi-center international trial also therefore aims to investigate different strategies that could be implemented in order to increase adherence and compliance to these prescribed exercise interventions in a long-term unsupervised environment. Although having 1-3 sessions performed unsupervised out of the 3-7 sessions required during the 16-week supervised phase of this trial could be considered a limitation of the study, it could also be identified as a strategy to promote adherence and compliance in a 'real-world setting'. Having specific guidance on what activities are available to implement the prescribed format of exercise (1-AIT, 4AIT, and MICT) in their own everyday environment, and how to monitor the intensity of training on a weekly basis for the initial 16-weeks could play a huge factor on their motivation and ability to perform the exercise program accurately and safely during the unsupervised phase. Another strategy being enforced in this trial is the requirement of participants to attend a supervised training session each month or every 6-months until the 1-year and 3-year end points, respectively, during the unsupervised phase. If these strategies proves to be effective in increasing both adherence and compliance of participants to the target intervention, then governing bodies around the globe should consider investing on providing vulnerable individuals with exercise-related services incorporated in this trial, such as the initial 16-week consultation with an exercise professional, followed-up with either a monthly/6-monthly visit with an exercise professional, or the use of physical activity and heart rate monitoring devices. This multi-center international trial indeed aims to ease the burden in healthcare/economic cost arising from treating end-stage CVD related conditions such as stroke and myocardial infarction, that could eventually emerge from the metabolic syndrome condition [27].

\begin{abstract}
Abbreviations
ACSM: American college of sports medicine; AIT: Aerobic interval training: CRP: C-reactive protein; CVD: Cardiovascular disease; EX-MET: Exercise in prevention of metabolic syndrome; $\mathrm{HbA}_{1}$ : Glycosylated hemoglobin; HDL: High-density lipoprotein; HR peak: Peak heart rate; IDF: International diabetes federation; MICT: Moderate-intensity continuous training; RPE: Rate of perceived exertion; TG: Triglycerides
\end{abstract}

\section{Acknowledgements}

The ergospirometry testing at NTNU was provided by NeXt Move, Norwegian University of Science and Technology (NTNU). NeXt Move is funded by the Faculty of Medicine and Health at NTNU and Central Norway Regional Health Authority.

\section{Funding}

The study received two peer - review funding's from the Joint Research Committee between St. Olavs Hospital and the Faculty of Medicine and Health sciences, Norwegian University of Science and Technology (NTNU).

Availability of data and materials

Not applicable

\section{Authors' contributions}

AET and JSR wrote the manuscript, reviewed/edited and approved the final draft of the manuscript. UW, AET, and JSC designed the study. JSR, KJ, MH, $A P, E E, \varnothing S, J R, P S M, J C$, and CRBJ also substantially contributed to the conception and design of the study, and reviewed/edited and approved the final draft of the manuscript

\section{Ethics approval and consent to participate}

EX-MET has been approved by the Regional Committee for Medical Research Ethics (REK 2011/2150). Oral and written informed consent is obtained from all participants before inclusion. Participation in the main study is not influenced by willingness to participate in sub-studies. The following local committees has also approved the study:

Ethical committee at School of Physical Education and Sport of Ribeirao Preto (University of Sao Paulo)

The University of Queensland Human Research Ethics Committee.

Comite de etica e investigacion en seres humandos del grupo hospitalario Kennedy Guayaquil - Ecuador.

The ethical board of the Faculty of Medicine, Technical University of Munich. This study protocol does not report any results from this study, but the protocol and standard operating procedure are available.

A steering committee has been appointed to dictate resolutions for major scientific and ethical questions. This committee developed the study protocol and is responsible for data collection, management, and publications derived from this study.

Status of this study today are active but not recruiting.

\section{Consent for publication}

Not applicable

Competing interests

There are no disclosures to report any competing interests.

\section{Publisher's Note}

Springer Nature remains neutral with regard to jurisdictional claims in published maps and institutional affiliations.

\section{Author details}

${ }^{1}$ K.G. Jebsen Center of Exercise in Medicine at Department of Circulation and Medical Imaging, Faculty of Medicine, Norwegian University of Science and Technology, Trondheim, Norway. ${ }^{2}$ School of Human Movement and Nutrition Sciences, University of Queensland, Brisbane, Queensland, Australia. ${ }^{3}$ Department of Prevention, Rehabilitation and Sports Medicine, Klinikum rechts der Isar, Technische Universität München, Munich, Germany. ${ }^{4} \mathrm{KJ}$ Fisiosport, Guayaquil, Ecuador. ${ }^{5}$ Department of Public Health and General 
Practice, Norwegian University of Science and Technology, Trondheim, Norway. ${ }^{6}$ School of Physical Education and Sport of Ribeirao Preto, University of São Paulo, Ribeiro Preto, Brazil. 'Sørlandet Hospital HF, Kristiansand, Norway. ${ }^{8}$ DZHK (German Center for Cardiovascular Research), partner site Munich Heart Alliance, Munich, Germany. ${ }^{9}$ Clinic of Cardiology, St. Olavs Hospital, Trondheim, Norway. ${ }^{10}$ Department of Circulation and Medical Imaging, Faculty of Medicine, Norwegian University of Science and Technology, Prinsesse Kristinas gt.3, 7006 Trondheim, Norway.

\section{Received: 23 October 2017 Accepted: 20 March 2018}

\section{Published online: 02 April 2018}

\section{References}

1. Katzmarzyk PT, Church TS, Janssen I, Ross R, Blair SN. Metabolic syndrome, obesity, and mortality: impact of cardiorespiratory fitness. Diabetes Care. 2005;28(2):391-7.

2. Katzmarzyk PT, Church TS, Blair SN. Cardiorespiratory fitness attenuates the effects of the metabolic syndrome on all-cause and cardiovascular disease mortality in men. Arch Intern Med. 2004;164(10):1092-7.

3. The IDF consensus worldwide definition of the metabolic syndrome [https://www.idf.org/our-activities/advocacy-awareness/resources-and-tools/60: idfconsensus-worldwide-definitionof-the-metabolic-syndrome.html].

4. Ford ES, Giles WH, Dietz WH. Prevalence of the metabolic syndrome among US adults: findings from the third National Health and nutrition examination survey. JAMA. 2002;287(3):356-9.

5. James PT, Rigby N, Leach R. The obesity epidemic, metabolic syndrome and future prevention strategies. Eur J Cardiovasc Prev Rehabil. 2004;11(1):3-8.

6. Balkau B, Charles MA, Drivsholm T, Borch-Johnsen K, Wareham N, Yudkin JS, Morris R, Zavaroni I, van Dam R, Feskins E, et al. Frequency of the WHO metabolic syndrome in European cohorts, and an alternative definition of an insulin resistance syndrome. Diabetes Metab. 2002;28(5):364-76.

7. Gami AS, Witt BJ, Howard DE, Erwin PJ, Gami LA, Somers VK, Montori VM. Metabolic syndrome and risk of incident cardiovascular events and death: a systematic review and meta-analysis of longitudinal studies. J Am Coll Cardiol. 2007:49(4):403-14.

8. Malik S, Wong ND, Franklin SS, Kamath TV, L'Italien GJ, Pio JR, Williams GR. Impact of the metabolic syndrome on mortality from coronary heart disease, cardiovascular disease, and all causes in United States adults. Circulation. 2004; 110(10):1245-50.

9. Pattyn N, Cornelissen VA, Eshghi SR, Vanhees L. The effect of exercise on the cardiovascular risk factors constituting the metabolic syndrome: a metaanalysis of controlled trials. Sports Med. 2013;43(2):121-33.

10. Mittleman MA, Maclure M, Tofler GH, Sherwood JB, Goldberg RJ, Muller JE. Triggering of acute myocardial infarction by heavy physical exertion. Protection against triggering by regular exertion. Determinants of myocardial infarction onset study investigators. N Engl J Med. 1993;329(23):1677-83.

11. Wisloff U, Stoylen A, Loennechen JP, Bruvold M, Rognmo O, Haram PM, Tjonna AE, Helgerud J, Slordahl SA, Lee SJ, et al. Superior cardiovascular effect of aerobic interval training versus moderate continuous training in heart failure patients: a randomized study. Circulation. 2007;115(24):3086-94.

12. Gibbons RJ, Balady GJ, Beasley JW, Bricker JT, Duvernoy WF, Froelicher VF, Mark DB, Marwick TH, McCallister BD, Thompson PD Jr, et al. ACC/AHA guidelines for exercise testing. A report of the American College of Cardiology/American Heart Association task force on practice guidelines (committee on exercise testing). J Am Coll Cardiol. 1997:30(1):260-311.

13. Center HR: Questionnaires 2006-2008. https:/www.ntnu.edu/hunt/data/que.

14. Bertheussen GF, Romundstad PR, Landmark T, Kaasa S, Dale O, Helbostad JL. Associations between physical activity and physical and mental health-a HUNT 3 study. Med Sci Sports Exerc. 2011;43(7):1220-8.

15. Landmark T, Romundstad P, Dale O, Borchgrevink PC, Kaasa S. Estimating the prevalence of chronic pain: validation of recall against longitudinal reporting (the HUNT pain study). Pain. 2012;153(7):1368-73.

16. Tjonna AE, Lee SJ, Rognmo O, Stolen TO, Bye A, Haram PM, Loennechen JP, Al-Share QY, Skogvoll E, Slordahl SA, et al. Aerobic interval training versus continuous moderate exercise as a treatment for the metabolic syndrome: a pilot study. Circulation. 2008;118(4):346-54.

17. Balducci S, Zanuso S, Nicolucci A, De Feo P, Cavallo S, Cardelli P, Fallucca S, Alessi E, Fallucca F, Pugliese G. Effect of an intensive exercise intervention strategy on modifiable cardiovascular risk factors in subjects with type 2 diabetes mellitus: a randomized controlled trial: the Italian diabetes and exercise study (IDES). Arch Intern Med. 2010;170(20):1794-803.
18. Borg G, Ljunggren G, Ceci R. The increase of perceived exertion, aches and pain in the legs, heart rate and blood lactate during exercise on a bicycle ergometer. Eur J Appl Physiol Occup Physiol. 1985;54(4):343-9.

19. Rognmo O, Hetland E, Helgerud J, Hoff J, Slordahl SA. High intensity aerobic interval exercise is superior to moderate intensity exercise for increasing aerobic capacity in patients with coronary artery disease. Eur I Cardiovasc Prev Rehabil. 2004;11(3):216-22.

20. Schjerve IE, Tyldum GA, Tjonna AE, Stolen T, Loennechen JP, Hansen HE, Haram PM, Heinrich G, Bye A, Najjar SM, et al. Both aerobic endurance and strength training programmes improve cardiovascular health in obese adults. Clin Sci (Lond). 2008;115(9):283-93.

21. Weston KS, Wisloff U, Coombes JS. High-intensity interval training in patients with lifestyle-induced cardiometabolic disease: a systematic review and meta-analysis. Br J Sports Med. 2014;48(16):1227-34.

22. Ramos JS, Dalleck LC, Tjonna AE, Beetham KS, Coombes JS. The impact of high-intensity interval training versus moderate-intensity continuous training on vascular function: a systematic review and meta-analysis. Sports Med. 2015;45(5):679-92.

23. Tjonna AE, Leinan IM, Bartnes AT, Jenssen BM, Gibala MJ, Winett RA, Wisloff $U$. Low- and high-volume of intensive endurance training significantly improves maximal oxygen uptake after 10-weeks of training in healthy men. PLoS One. 2013;8(5):e65382.

24. Blair SN, Kampert JB, Kohl HW 3rd, Barlow CE, Macera CA, Paffenbarger RS $\mathrm{Jr}$, Gibbons LW. Influences of cardiorespiratory fitness and other precursors on cardiovascular disease and all-cause mortality in men and women. JAMA. 1996:276(3):205-10.

25. Trost SG, Owen N, Bauman AE, Sallis JF, Brown W. Correlates of adults' participation in physical activity: review and update. Med Sci Sports Exerc. 2002;34(12):1996-2001

26. Kessler HS, Sisson SB, Short KR. The potential for high-intensity interval training to reduce cardiometabolic disease risk. Sports Med. 2012;42(6):489-509.

27. Galassi A, Reynolds K, He J. Metabolic syndrome and risk of cardiovascular disease: a meta-analysis. Am J Med. 2006;119(10):812-9.

\section{Submit your next manuscript to BioMed Central and we will help you at every step:}

- We accept pre-submission inquiries

- Our selector tool helps you to find the most relevant journal

- We provide round the clock customer support

- Convenient online submission

- Thorough peer review

- Inclusion in PubMed and all major indexing services

- Maximum visibility for your research

Submit your manuscript at www.biomedcentral.com/submit
) Biomed Central 